\title{
RBP4 functions as a hepatokine in the regulation of glucose metabolism by the circadian clock in mice
}

\author{
Xiang $\mathrm{Ma}^{1,2} \cdot \operatorname{Zan} \mathrm{Zhou}^{1,2} \cdot$ Yaqiong Chen ${ }^{1} \cdot$ Yuting $\mathrm{Wu}^{1} \cdot$ Yi Liu ${ }^{1}$
}

Received: 6 August 2015 / Accepted: 22 October 2015 / Published online: 13 November 2015

(C) Springer-Verlag Berlin Heidelberg 2015

\begin{abstract}
Aims/hypothesis As one of the key adipokines, retinol binding protein 4 (RBP4) is suggested to positively correlate with insulin resistance; however, not all clinical studies support this association. Although some explanations are proposed for this discrepancy, the temporal aspect of RBP4 secretion has not been considered. Aryl hydrocarbon receptor nuclear translocator-like (also known as BMAL1) and its target $\mathrm{D}$ site-binding protein (DBP) are both pivotal transcription factors of the circadian core clock. Given the overwhelming presence of circadian control in metabolism and the principal role of the liver in RBP4 secretion, we hypothesised that RBP4 may oscillate under the control of BMAL1 and act as a hepatokine, participating in the maintenance of glucose homeostasis by the circadian clock.

Methods We used liver-specific Bmall (also known as Arntl)knockout mice and recombinant adenoviruses expressing short-hairpin RNA (shRNA) specific for Dbp or Rbp4 in the liver.
\end{abstract}

Xiang Ma and Zan Zhou contributed equally to this work.

Electronic supplementary material The online version of this article (doi:10.1007/s00125-015-3807-1) contains peer-reviewed but unedited supplementary material, which is available to authorised users.

Yi Liu

liuyi@sibs.ac.cn

1 Key Laboratory of Nutrition and Metabolism, Institute for Nutritional Sciences, Shanghai Institutes for Biological Sciences, Chinese Academy of Sciences, 320 Yueyang Road, Shanghai 200031, People's Republic of China

2 Shanghai Institutes for Biological Sciences, University of the Chinese Academy of Sciences, Shanghai, People's Republic of China
Results RBP4 displayed diurnal oscillations in the liver and plasma, which were dampened in liver-specific-Bmall-knockout mice. BMAL1 regulated hepatic RBP4 expression via its direct target, DBP. Hepatic knockdown of RBP4 or DBP increased whole-body insulin sensitivity in mice in a time-of-daydependent manner. Conversely, hepatic overexpression of RBP4 reversed the insulin-sensitising effects of liver-specific depletion of BMAL1.

Conclusions/interpretation Our results not only provide a novel mechanism for circadian regulation of RBP4, but also unveil a critical role of RBP4, acting as a hepatokine in the regulation of glucose metabolism by the circadian clock.

Keywords BMAL1 - Circadian clock · DBP - Glucose metabolism $\cdot$ Insulin sensitivity $\cdot$ RBP4

\begin{tabular}{|c|c|}
\hline \multicolumn{2}{|c|}{ Abbreviations } \\
\hline BMAL1 & $\begin{array}{l}\text { Aryl hydrocarbon receptor } \\
\text { nuclear translocator-like }\end{array}$ \\
\hline ChIP & Chromatin immunoprecipitation \\
\hline CLOCK & Clock circadian regulator \\
\hline CREB & $\begin{array}{l}\text { cAMP responsive element binding } \\
\text { protein } 1\end{array}$ \\
\hline DBP & D site-binding protein \\
\hline DBS & DBP-binding site \\
\hline G6pase & Glucose-6-phosphatase \\
\hline GFP & Green fluorescent protein \\
\hline${\mathrm{L}-\mathrm{Bmal1}^{-/-}}^{-1}$ & Liver-specific deletion of Bmall \\
\hline PCK1 & $\begin{array}{l}\text { Phosphoenolpyruvate } \\
\text { carboxykinase } 1 \text {, cytosolic }\end{array}$ \\
\hline RBP4 & Retinol binding protein 4 \\
\hline $\mathrm{RBP} 4-\Delta 18$ & $\begin{array}{l}\text { Retinol binding protein } 4 \text { without } \\
\text { signal peptide }\end{array}$ \\
\hline Rbp4-Luc & Rbp4-luciferase \\
\hline
\end{tabular}




\begin{tabular}{|c|c|}
\hline $\begin{array}{l}\text { RNAi } \\
\text { shRNA }\end{array}$ & $\begin{array}{l}\text { RNA interference } \\
\text { short-hairpin RNA }\end{array}$ \\
\hline SIBS & $\begin{array}{l}\text { Shanghai Institutes for } \\
\text { Biological Sciences }\end{array}$ \\
\hline USi & Unspecific RNAi \\
\hline NT & Wild-type \\
\hline ZT & Zeitgeber time \\
\hline
\end{tabular}

\section{Introduction}

Retinol binding protein 4 (RBP4), a specific carrier for retinol in the circulation [1], is produced and released mainly by hepatocytes (80\%) [2] and adipocytes (20\%) [3]. A previous study has reported that elevation of adipocyte-secreted RBP4 contributes to the development of insulin resistance in mice [4]. Clinical studies have shown that circulating RBP4 levels are increased in individuals with impaired glucose tolerance and type 2 diabetes, and correlate inversely with insulin sensitivity [4-7]. However, these associations are not supported by all studies in human, as several studies found no correlation between circulating RBP4 levels and insulin resistance, impaired glucose tolerance or type 2 diabetes [8-12]. This discrepancy suggests a limited understanding of the relationship between RBP4 and insulin resistance. Although liver is the principal source of circulating RBP4 [2], it remains unknown whether hepatic RBP4 plays a role in modulating glucose homeostasis.

The circadian clock is an intrinsic time-keeping system that enables organisms to adapt their physiological and behavioural rhythms in response to the external environment [13]. While the light-responsive central clock resides in the suprachiasmatic nucleus, individual clocks are present in the peripheral tissues [14-17]. The current molecular model for the circadian clock is based on a transcriptional feedback loop in which aryl hydrocarbon receptor nuclear translocator-like (BMAL1)/clock circadian regulator (CLOCK) heterodimers drive the transcription of cryptochrome 1 and 2 (Cryl and Cry2) and period circadian clock 1, 2 and 3 (Per1, Per2 and Per3) by binding to the E-boxes on their promoters. In turn, the cryptochrome (CER)/period (PER) complex represses the transcriptional activity of BMAL1/ CLOCK, which generates intrinsically oscillatory signals [18-20]. This core clock machinery regulates the rhythmic expression of downstream genes, largely by coordinating with other transcription factors, including their direct targets (e.g. D site-binding protein (Dbp), Ppara [also known as Ppara], and Ppary [also known as Parg]) [19]. However, little is known about how these clock components influence RBP4 in the liver and plasma.

While recent studies strongly suggest an important role of circadian clocks in glucose homeostasis [21, 22], the underlying mechanism remains to be elucidated. Thus, we hypothesised that the levels of Rbp4 mRNA or RBP4 protein would display diurnal oscillations that would be regulated by BMAL1, and that RBP4 may be required for the regulation of glucose metabolism by the circadian clock. With this in mind, we studied liver-specific-Bmal-knockout mice, a circadian-clock-disruption mouse model that has an insulin-sensitive phenotype. In addition, we took advantage of adenoviruses that selectively inhibit the expression of $D b p$ or $R b p 4$ in the mouse liver.

\section{Methods}

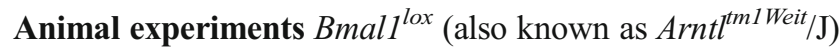
were purchased from the Jackson Laboratory (Bar Harbor, ME, USA). Bmal1 ${ }^{l o x}$ mice were mated with the C57BL/6 J strain expressing a Cre-recombinase to generate mice with liverspecific deletion of Bmall (L-Bmal1 ${ }^{-1}$ ), as described by Lamia et al [21]. $\mathrm{RBP}^{-/-}$mice were generated on a C57BL/6 J-genetic background by SIDANSAI Biotechnology (Shanghai, China) using transcription activator-like effector nuclease (TALEN)mediated gene targeting, as described by Chen et al [23]. The 8- to 10-week-old male C57BL/6 mice were purchased from Shanghai Laboratory Animal Centre (Shanghai, China). All mice were housed and bred in the animal facility at Shanghai Institutes for Biological Sciences (SIBS) at $22 \pm 2^{\circ} \mathrm{C}$, maintained on a $12 \mathrm{~h} / 12 \mathrm{~h}$ light/dark cycle and fed ad libitum for at least 2 weeks before the study. Recombinant adenovirus was delivered by systemic tail-vein injection to mice. At 10-25 days after viral infection, mice were subjected to glucose and insulin tolerance tests. See electronic supplementary material (ESM) Methods for further details. All animal care and use procedures were in accordance with guidelines of the Institutional Animal Care and Use Committee of the Institute for Nutritional Sciences (INS), SIBS and the Chinese Academy of Sciences (CAS).

Plasmids Full-length mouse cDNAs, Rbp4-luciferase (Rbp4-Luc) and shRNA plasmid constructions were cloned into the vectors indicated using standard molecular biology techniques. See ESM Methods for details. For cloning primers and oligonucleotides, see ESM Tables 1 and 2.

Adenoviruses and adeno-associated viruses Adenoviruses encoding RBP4, retinol binding protein 4 without signal peptide (RBP4- $\Delta 18$ ), control green fluorescent protein (GFP), DBP, BMAL1, with Rbp4 RNA interference (RNAi), $D b p$ RNAi, Bmall RNAi and control unspecific RNAi adenoviruses were generated through homologous recombination between a linearised transfer vector pAD-Track and the adenoviral backbone vector pAD-Easy, as described by $\mathrm{He}$ et al [24]. Viruses were purified by the $\mathrm{CsCl}$ method and dialysed against PBS buffer containing 10\% glycerol as described by Becker et al [25]. The Rbp4 RNAi adeno-associated virus was generated by Obio Technology (Shanghai, China). 
Culture of primary hepatocytes Primary hepatocytes were prepared from mice by collagenase perfusion techniques. See ESM Methods for further details.

Quantitative RT-PCR Quantitative RT-PCR was used to determine the relative expression levels of mRNAs. See ESM Methods and ESM Table 3 for further details.

Antibodies and western blot Anti-BMAL1 (ab3350) and anti-DBP (ab22824) were obtained from Abcam (Cambridge, UK). Cellular RBP4 (2947-1) was purchased from Epitomics (Burlingame, CA, USA) and plasma RBP4 antibody was a gift from Y. Liu (Institutes for Nutritional Sciences, Shanghai, China). Tissue and cell proteins were lysed in hypotonic buffer ( $1 \%$ Nonidet P-40, 50 mmol/l Tris-HCl, $0.1 \mathrm{mmol} / 1$ EDTA, $150 \mathrm{mmol} / \mathrm{l} \mathrm{NaCl}, \mathrm{pH}$ 7.4). See ESM Methods and ESM Table 4 for further details.

Chromatin immunoprecipitation Chromatin immunoprecipitation assays were done as described by Sun et al [26]. See ESM Methods for further details. The primer sequences are shown in ESM Table 5.

Generation of reporter cell lines Reporter cells and clonal lines were generated as previously described [27]. See ESM Methods for further details.

Bioluminescence recording and data analysis A LumiCycle luminometer (LumiCycle 32, Actimetrics, Wilmette, IL, USA) was used for bioluminescence recording. The LumiCycle Analysis program version 2.53 (Actimetrics, Wilmette, IL, USA) was used to determine circadian variables, as described elsewhere [28].

Luciferase assay The luciferase reporter assay for HEK293T cells has been described previously [29].

Statistical analysis Results are shown as mean \pm SEM. Statistical analysis was performed using one-way or twoway ANOVA or two-tailed unpaired Student's $t$ test where appropriate. Differences were considered statistically significant at $p<0.05$. All experiments were performed on at least two independent occasions.

\section{Results}

Hepatic BMAL1 deficiency dampens Rbp4 expression To explore the daily changes in $R b p 4$ expression, we analysed the mRNA levels of Rbp 4 and Bmall in the livers of mice killed at $4 \mathrm{~h}$ intervals around the clock (Fig. 1a, b). The accumulation of Rbp 4 mRNA and RBP4 protein displayed robust diurnal rhythms in the livers of wild-type (WT) mice, peaking around
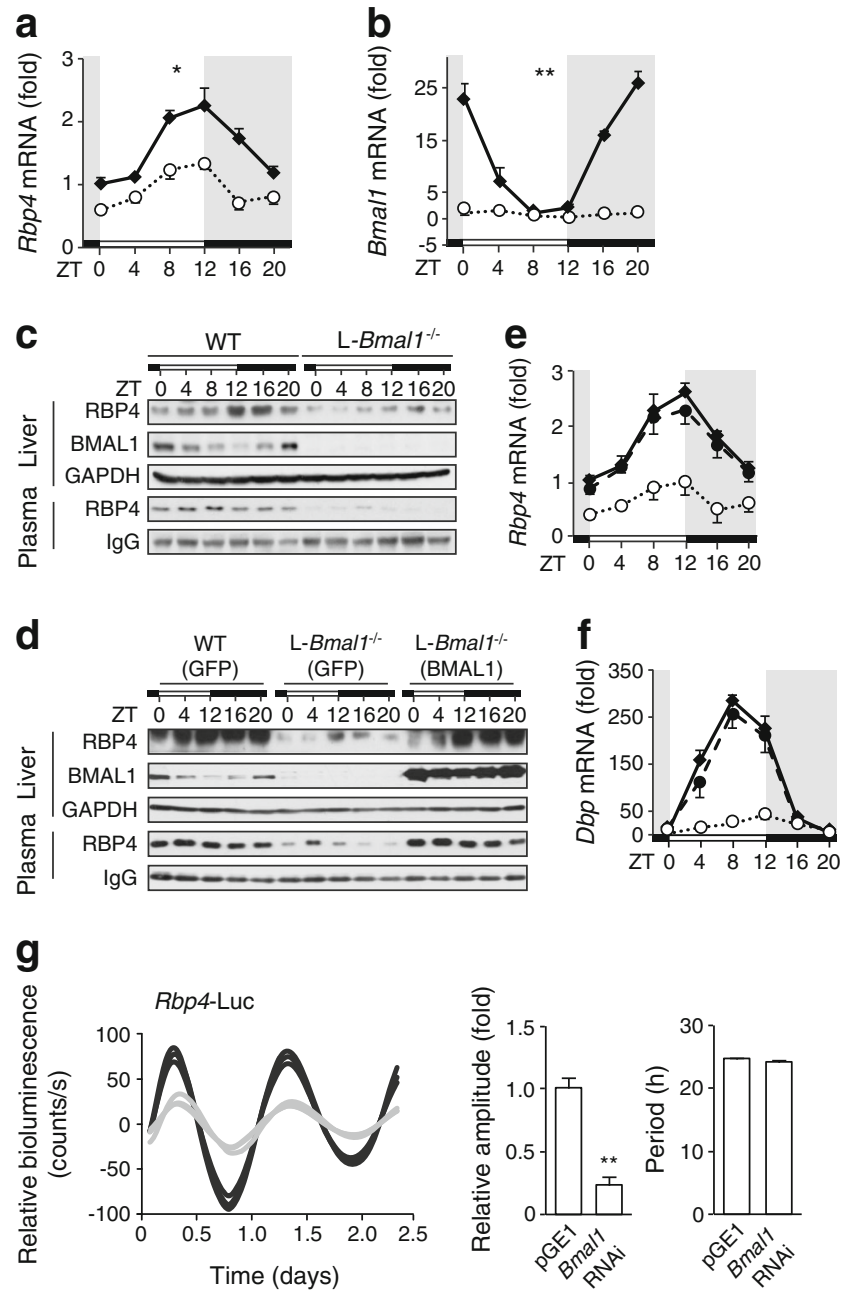

Fig. 1 BMAL1 regulates the rhythmic oscillation of Rbp4 in the liver. L-Bmall ${ }^{-1}$ and WT littermate mice were killed at $4 \mathrm{~h}$ intervals across the $24 \mathrm{~h}$ light/dark cycle. Quantitative PCR analysis of relative mRNA levels of (a) Rbp4 and (b) Bmall in the liver. Values were normalised to Gapdh and are presented relative to the lowest WT control, set at 1. Black diamonds, WT; white circles, L-Bmall ${ }^{-/}$. Grey shading and dark bars indicate dark periods $(n=3-4) .{ }^{*} p<0.05$ and ${ }^{* *} p<0.01$ with two-way ANOVA. (c) Immunoblotting, with the antibodies indicated, of liver homogenates or plasma at the ZTs indicated. WT (injected with Ad-gfp), L-Bmall ${ }^{-/-}$(injected with Ad-gfp) and L-Bmall ${ }^{-/-}$(injected with Ad-Bmall) mice were killed at $4 \mathrm{~h}$ intervals across the $24 \mathrm{~h}$ light/dark cycle. (d) Immunoblotting, with the antibodies indicated, of liver homogenates or plasma at the ZTs indicated. $(n=3-4)$. Quantitative PCR analysis of relative (e) $R b p 4$ and (f) $D b p$ mRNA levels in the livers. Black diamonds, WT (GFP); white circles, L-Bmall ${ }^{-1-}$ (GFP); black circles, L-Bmal1 ${ }^{-1-}$ (BMAL1). (g) Bioluminescence rhythm of Rbp4-Luc collected from NIH3T3 cells transfected with pGE1 (GeneErase shRNA expression vector, see ESM Methods for further details) or Bmall RNAi. Period and relative amplitude are presented. $n=3$; black lines, pGE1; grey lines, Bmall RNAi . ${ }^{* *} p<0.01$ with Student's $t$ test. Data are presented as mean \pm SEM

the onset of night (zeitgeber time [ZT] 12, Fig. 1a, c). Interestingly, plasma RBP4 levels also oscillated across the $24 \mathrm{~h}$ light/dark cycle, but with an $8 \mathrm{~h}$ phase advance compared with its hepatic expression (Fig. 1c). Furthermore, liver-specific 
knockout of Bmall significantly attenuated the amplitude of Rbp 4 mRNA and protein rhythms in the liver and plasma without altering their phases (Fig. 1a-c). In contrast, the oscillation of Rbp4 mRNA in white adipose tissue was little affected in L-Bmall ${ }^{-/-}$mice (ESM Fig 1a, b). Moreover, the damped oscillation of RBP4 in L-Bmall ${ }^{-/}$mice was restored to full-blown rhythmicity in the liver and plasma by ectopic expression of hepatic BMAL1 (Fig. 1d-f). Consistently, Bmall RNAi-mediated knockdown markedly attenuated the amplitude of the oscillation of Rbp4-Luc in NIH3T3 cells, with little effect on its period as monitored by real-time bioluminescence imaging (Fig. 1g). However, to our surprise, the results of the chromatin immunoprecipitation (ChIP) assay showed no presence of BMAL1 on the Rbp4 promoter (ESM Fig. 1c, d).

DBP transactivates $R b p 4$ in the liver Further analysis of the Rbp 4 promoter sequence showed homology of the nucleotide sequence located from -132 to -809 in the $R b p 4$ gene with the consensus DBP-binding site (DBS; ESM Fig. 2a) required for the recruitment of transcription factor DBP; hepatic expression of the DBP was abolished in L-Bmal1 ${ }^{-/}$mice (ESM Fig. 2b). We then tested the ability of DBP to drive $R b p 4$ transcription through these putative DBSs. Overexpression of DBP significantly stimulated Rbp4-Luc activity in a dose-dependent manner (Fig. 2a), whereas deletion of the putative DBSs abolished DBP-induced activity (Fig. 2b). ChIP analysis confirmed the presence of DBP on these DBSs in the Rbp 4 promoter (Fig. 2c). Consistently, we observed that the amplitude of the oscillation of Rbp4-Luc was greatly attenuated by $D b p$ RNAi (ESM Fig. 2c) in NIH3T3 cells by using real-time bioluminescence imaging. Moreover, adenoviral expression of $D b p$ (Ad- $D b p$ ) significantly enhanced the expression of RBP4 in primary hepatocytes. Conversely, $D b p$ RNAi adenovirus (Ad-Dbpi) decreased Rbp 4 mRNA levels, which led to a reduction in levels of both expression and secretion of RBP4 protein in primary hepatocytes (ESM Fig. 2d-f). In vivo investigation revealed that Ad-Dbpi significantly decreased the amplitude of RBP4 protein and mRNA oscillation in mouse liver (Fig. 2d-f). Furthermore, adenoviral overexpression of Bmall or Dbp was sufficient to restore both the levels and secretion of RBP4 in $\mathrm{Bmal}^{\prime-}$ primary hepatocytes, suggesting that DBP is involved in the mechanism by which BMAL1 regulates RBP4 (Fig. 2g).

Hepatic RBP4 modulates glucose metabolism To determine the effects of hepatocyte-derived RBP4 in regulating glucose homeostasis, we prepared an Rbp4 RNAi adenovirus (Ad-Rbp4i), which specifically reduced Rbp4 expression levels in the liver but not in white adipose tissue (ESM Fig. 3a,b). Compared with mice expressing unspecific RNAi (Ad-USi), levels of resting glucose (the blood glucose level in mice fed ad libitum) were significantly lower in RBP4-knockdown mice
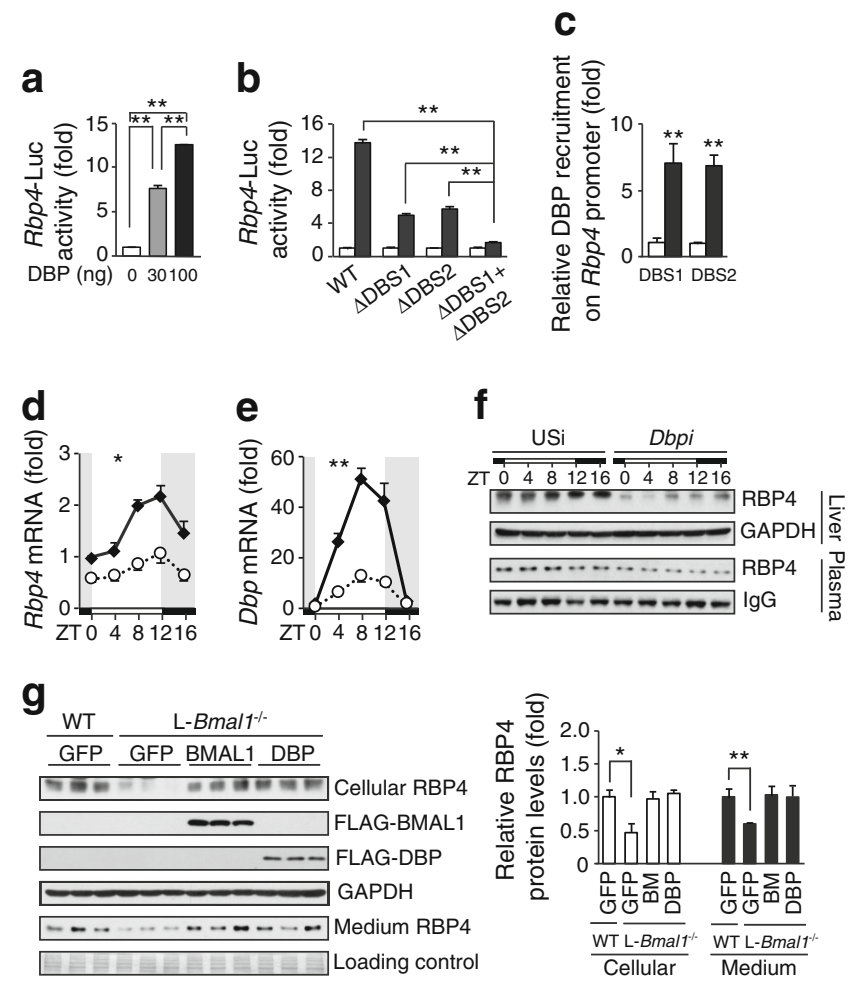

Fig. $2 R b p 4$ transcription is regulated by DBP in the liver. (a) Relative luciferase activity of Rbp4-Luc in the presence of $30 \mathrm{ng}$ and $100 \mathrm{ng}$ doses of DBP in 293 T cells. Values were normalised to $\beta$-gal and are presented relative to control, set at $1 ;{ }^{*} p<0.01$ with Student's $t$ test. (b) Relative luciferase activity of Rbp4-promoter mutants in the presence of vector expressing DBP in 293 T cells. WT, wild-type Rbp4-promoter; $\triangle \mathrm{DBS} 1$ and $\triangle \mathrm{DBS} 2, R b p 4$ promoter with a deletion of the putative DBP-binding site 1 (ATTATGTTTT) and 2 (GTTACGAAAG); respectively. White bars, plasmid cytomegalovirus promoter (pc)DNA; black bars, DBP; ${ }^{* *} p<0.01$ with Student's $t$ test. (c) ChIP analysis showing the relative recruitment of DBP to DBS1 and DBS2 on the promoter of Rbp4 in mouse primary hepatocytes synchronised by dexamethasone $(1 \mu \mathrm{mol} / \mathrm{l})$ for $2 \mathrm{~h}$. White bars, anti-IgG; black bars, anti-DBP; $* * p<0.01$ with Student's $t$ test. Mice injected with Ad-USi or Ad-Dbpi adenovirus were killed at $4 \mathrm{~h}$ intervals across the $24 \mathrm{~h}$ light/dark cycle. Quantitative PCR analysis of relative mRNA levels of (d) $R b p 4$ and (e) $D b p$ in liver. Black diamonds, USi; white circles, Dbpi. Grey shading and dark bars indicate dark periods $(n=3-4) ;{ }^{*} p<0.05$ and ${ }^{* *} p<0.01$ with two-way ANOVA. (f) Immunoblotting, with the antibodies indicated, of liver homogenates or plasma at the ZTs indicated. (g) Immunoblotting analysis of RBP4 proteins in WT or Bmall-null primary hepatocytes infected with adenovirus expressing GFP, BMAL1 and DBP. Hepatocytes were synchronised as before. Relative protein levels of RBP4 are presented. Values are normalised to GAPDH or loading control and are presented relative to control, set at $1 ;{ }^{*} p<0.05$ and $* * p<0.01$ with Student's $t$ test. Data are presented as mean \pm SEM. GAPDH, glyceraldehyde 3-phosphate dehydrogenase

around most time points (ZT0, 4, 8, 20) except ZT12 and 16 (Fig. 3a). Consistently, Rbp 4i significantly increased insulin sensitivity in mice at ZT4, whereas these effects were not observed at ZT16 (Fig. 3b, c). These two sets of data indicate that RBP4 modulates glucose metabolism mainly during resting/fasting periods (daytime for mice) when hepatic gluconeogenesis is stimulated. Indeed, analysis of 

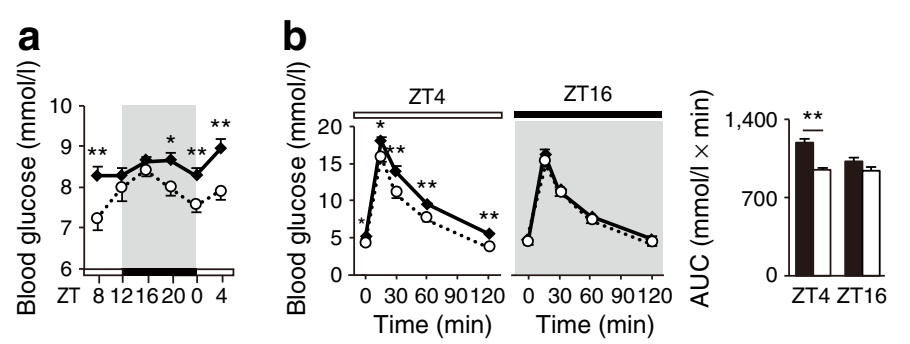

C

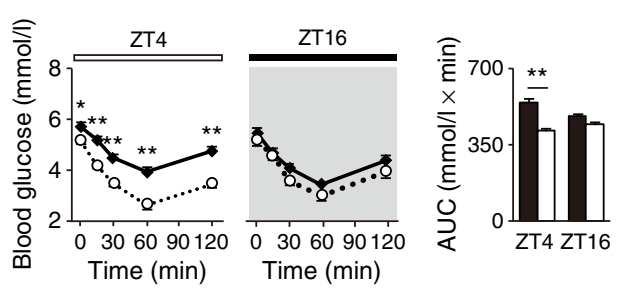

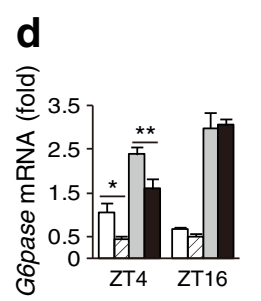
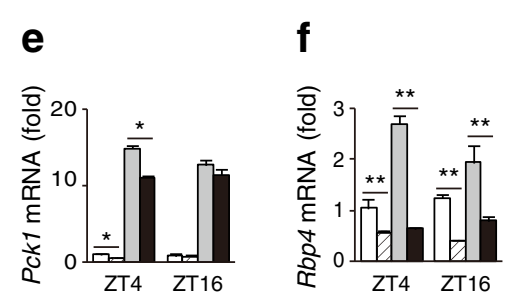

Fig. 3 Temporal effects of hepatic RBP4 knockdown on glucose metabolism. WT mice were injected with adenovirus expressing Rbp4i or USi control. (a) Resting glucose levels were measured around indicated ZTs $(n=12-14)$. (b) GTT and (c) ITT were performed around ZT4 and ZT16 $(n=12-14)$. Mice were fasted for $16 \mathrm{~h}$ before GTT and $2 \mathrm{~h}$ before ITT. AUCs were measured. Black diamonds and bars, USi; white circles and bars, Rbp $4 \mathrm{i} ; * p<0.05$ and ${ }^{* *} p<0.01$ with one-way ANOVA. (d-f) Quantitative PCR

gluconeogenic genes demonstrated that mRNA levels of $P c k 1$ and glucose 6-phosphatase (G6pase, also known as G6pc) as well as protein levels of phosphorylated cAMP responsive element binding protein 1 (CREB) and phosphoenolpyruvate carboxykinase 1, cytosolic (PCK1) were decreased by RBP4 knockdown around ZT4, but not ZT16 (Fig. 3d-g). We further confirmed these results by delivering $R b p 4 \mathrm{i}$ via an adenoassociated virus ( $R b 4 \mathrm{i})$, which caused very mild staining on the immunoblot (ESM Fig. 4).

To explore the possibility that intracellular hepatic RBP4 may be implicated in the regulation of glucose homeostasis, we prepared an adenovirus expressing $R b p 4$ with the deletion of secretory signal peptide (Ad-Rbp4- $\Delta 18)$. Injection of this virus significantly increased resting blood glucose levels in mice around ZT0 and ZT4, but had little effect during the rest of the day (ESM Fig. 5a). Consistently, glucose tolerance was impaired in mice expressing hepatic Ad-Rbp4- $\Delta 18$, compared with those injected with control GFP adenovirus, at around ZT0 (ESM Fig. 5b). In parallel with these results, the mRNA levels of gluconeogenic genes Pckl, Pgcl $\alpha$ (also known as Ppargcla) and Fbpl were also significantly increased in the livers of Ad-Rbp4- $\Delta 18$-injected mice at ZT0 (ESM Fig. 5c-f). Meanwhile, protein levels of PCK1 and phosphorylated CREB were increased by Ad-Rbp4- $\Delta 18$ in the liver without affecting the total level of CREB protein at ZT0 (ESM Fig. $5 \mathrm{~g}$ ). Importantly, injection of Ad-Rbp4- $\Delta 18$ led to significant accumulation of $R b p 4-\Delta 18$ within the liver, but had little effect on plasma RBP4 levels (ESM Fig. 5g).
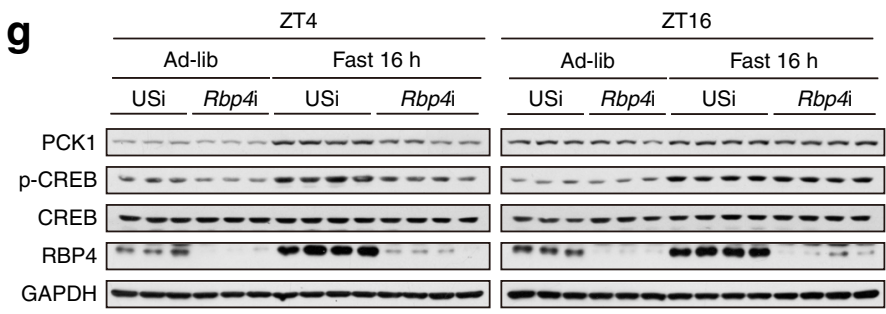

analysis of relative G6pase, Pck1 and Rbp4 mRNA levels in the liver. White bars, ad-libitum-fed USi; hatched bars, ad-libitum-fed Rbp4i mice; grey bars, fasted USi; black bars, fasted $R B P 4 \mathrm{i}$ mice; ${ }^{*} p<0.05$ and ${ }^{* *} p<0.01$ with Student's $t$ test. Data are presented as mean \pm SEM. (g) Immunoblotting of liver homogenates with the antibodies indicated. Mice were killed at ZT4 or ZT16, fed or fasted for $16 \mathrm{~h}(n=3-4)$. Values were normalised to Gapdh and are presented relative to fed control, set (ZT4) at 1. Ad-lib, ad-libitum-fed

DBP knockdown in the liver improves whole-body insulin sensitivity As hepatic RBP4 participates in the regulation of insulin sensitivity, we expected DBP to also be involved in glucose metabolism. In line with the effects of Rbp 4 i (Fig. 3), the injection of DBP-knockdown adenovirus (Dbpi) not only lowered blood glucose levels during the daytime in mice fed ad libitum but also ameliorated insulin sensitivity around ZT4 but not ZT16 (Fig. 4a-c). Consistently, Dbpi significantly reduced the protein and mRNA levels of gluconeogenic genes in livers from $16 \mathrm{~h}$ fasted mice and mice fed ad libitum collected around ZT4 but, in contrast, had little effects in those around ZT16 (Fig. 4d-h).

The involvement of RBP4 in BMAL1/DBP regulation of glucose homeostasis Based on our findings, we speculate that RBP4 is involved in DBP-regulated glucose metabolism. Indeed, transduction of DBP adenovirus significantly increased the amounts of PCK1 protein in primary hepatocytes derived from WT mice, but not in those from Rbp4-knockout mice (Fig. 5a). In addition, adenoviral expression of $R b p 4$ restored the reduction in PCK1 protein by Ad-Dbpi in primary hepatocytes (Fig. 5b). These observations prompted us to examine the role of RBP4 in meditating the effects of BMAL1 on glucose metabolism. Consistent with our previous study [21], L-Bmal1 ${ }^{-/}$mice had enhanced glucose tolerance compared with WT littermates during the daytime, together with decreased protein levels of G6Pase, PCK1 and phosphorylated CREB. However, hepatic expression of Rbp 4 by adenoviral 

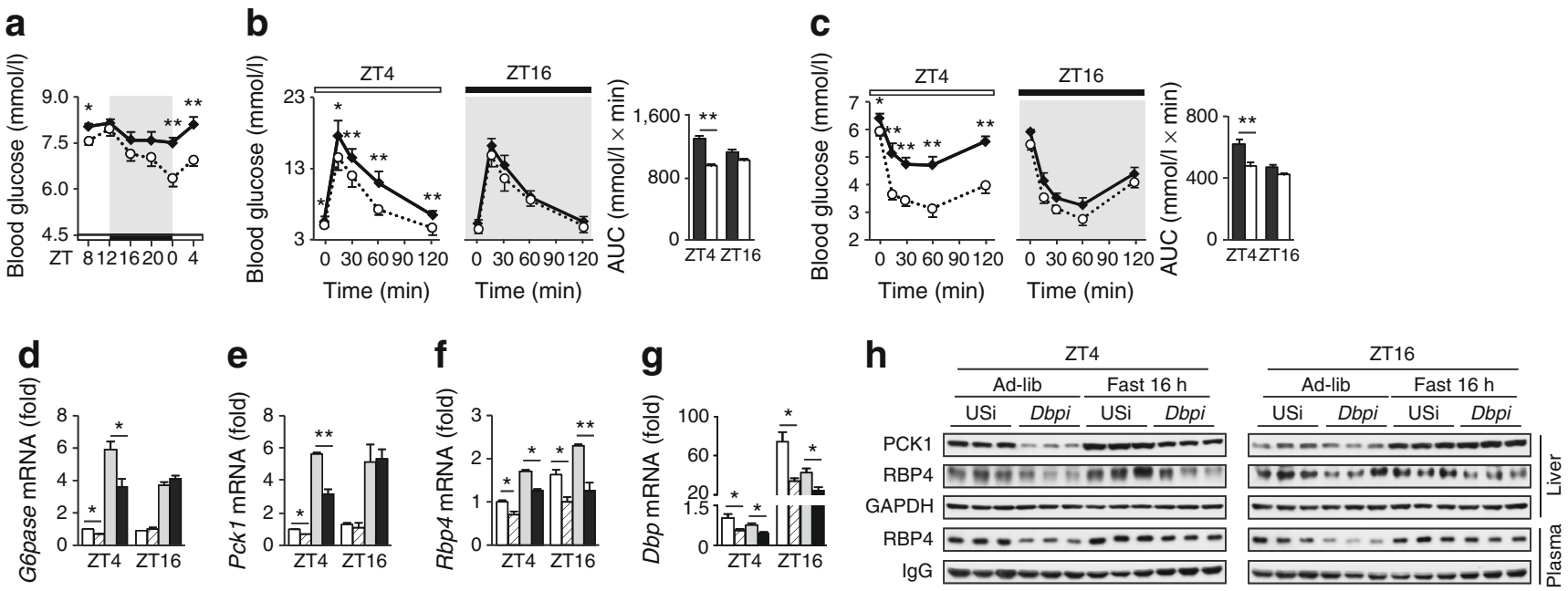

Fig. 4 Temporal effects of hepatic DBP knockdown on glucose metabolism. WT mice were injected with adenovirus expressing $D b p$ RNAi (Dbpi) or USi control. (a) Resting glucose levels were measured around the ZTs indicated $(n=10-12)$. (b) GTT and (c) ITT were performed around ZT4 and ZT16 $(n=8-10)$. Mice were fasted for $16 \mathrm{~h}$ before GTT and $2 \mathrm{~h}$ before ITT. AUCs were measured. (a-c) Black diamonds and bars, USi; white circles and bars, $D b p i$; ${ }^{*} p<0.05$ and ${ }^{* *} p<0.01$; oneway ANOVA. (d-g) Quantitative PCR analysis of relative G6pase, Pck1

$R b p 4$ and $D b p$ mRNA levels in the liver. White bars, ad-libitum-fed USi; hatched bars, ad-libitum-fed Dbpi; grey bars, fasted USi; black bars, fasted Dbpi; ${ }^{*} p<0.05$ and ${ }^{* *} p<0.01$ with Student's $t$ test. (h) Immunoblotting of the liver homogenates or plasma with the antibodies indicated. Mice were killed at ZT4 or ZT16, fed or fasted for $16 \mathrm{~h}(n=3)$. Values were normalised to Gapdh and are presented relative to fed control, set (ZT4) at 1 . Data are presented as mean \pm SEM. Ad-lib, ad-libitum-fed

delivery reversed such beneficial effects of L-Bmall deficiency in mice (Fig. 5c-e).

\section{Discussion}

Previous studies have revealed that a substantial fraction ( 10\%) of all liver mRNA is expressed in a rhythmic fashion, and many of the proteins are involved in glucose metabolism [30]. In this study, we found that RBP4 oscillates in a circadian manner throughout $24 \mathrm{~h} \mathrm{light/dark} \mathrm{cycles,} \mathrm{not} \mathrm{only} \mathrm{in} \mathrm{the} \mathrm{liver} \mathrm{and} \mathrm{plas-}$ ma, but also in the white adipose tissue. We also found that this diurnal variation is dampened in liver-specific Bmall-knockout mice. These results indicate a critical role of BMAL1 in the regulation of hepatic Rbp4 transcription, which determines its rhythm in the plasma. By using ChIP analysis and adenovirusmediated RNA interference, we provide a mechanism through which BMAL1 drives the circadian expression of hepatic RBP4 via DBP. The liver-enriched transcription factor DBP oscillates in a daily manner under the control of BMAL1 [31, 32]. In line with previous studies [32, 33], mRNA levels of $D b p$ peak around ZT8 (ESM Fig. 2b), which is compatible with the accumulation of Rbp 4 transcripts (Fig. 1a). Our results demonstrate that DBP is a novel transcriptional activator of RBP4 in the liver.

Interestingly, our data show that the rhythm of plasma RBP4 displays an $8 \mathrm{~h}$ phase advance compared with its hepatic expression (Fig. 1c). Based on the important role of diet in retinol supply, we speculate that this phase advance could be due to dietary retinol uptake control by feeding cycle. In contrast to the role of RBP4 in the transport of hepatic

retinol to plasma during resting/fasting periods (daytime for mice), postprandial retinol is mainly mobilised to peripheral tissues via chylomicrons. Therefore, the oscillation of serum RBP4 peaks in the daytime and reaches a nadir after eating (night-time), whereas hepatic RBP4 oscillates with a reversed phase, regulated by the circadian clock, which leads to the apparent $8 \mathrm{~h}$ phase lag between these two rhythms.

Although circulating RBP4 levels are consistently correlated with insulin resistance in mice [4, 34], clinical studies in human have not reached a consensus on this issue [8-12]. We have demonstrated that RBP4 displays an oscillatory pattern in the plasma (Fig. 1c), which provides an alternative interpretation for the proposed correlation. As the amount of RBP4 in the blood changes within a couple of hours, if the sampling time interval is too long or the sampling time points are different, the results could be conflicting, which is further supported by the fact that resting glucose levels are not altered in RBP4-knockdown mice around ZT12 and ZT16 (Fig. 3a). Meanwhile, insulin sensitivity is increased in RBP4-knockdown mice at ZT4, but remains unchanged at ZT16, which strongly implies an underlying circadian process in the regulation of glucose metabolism by RBP4 (Fig. 3b, c). Together, our studies emphasise the need to apply strict control to the timing of blood sampling and to consider circadian effects when interpreting results.

Compared with adipose tissue, the liver is the major site for the synthesis and secretion of RBP4 [1], as one hepatocyte expresses about five times as much $R b p 4$ mRNA as an adipocyte under either lean [35] or obese [36] conditions. 
a

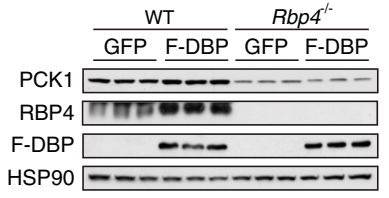

b
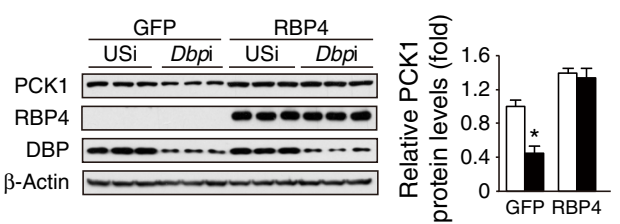

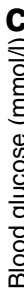
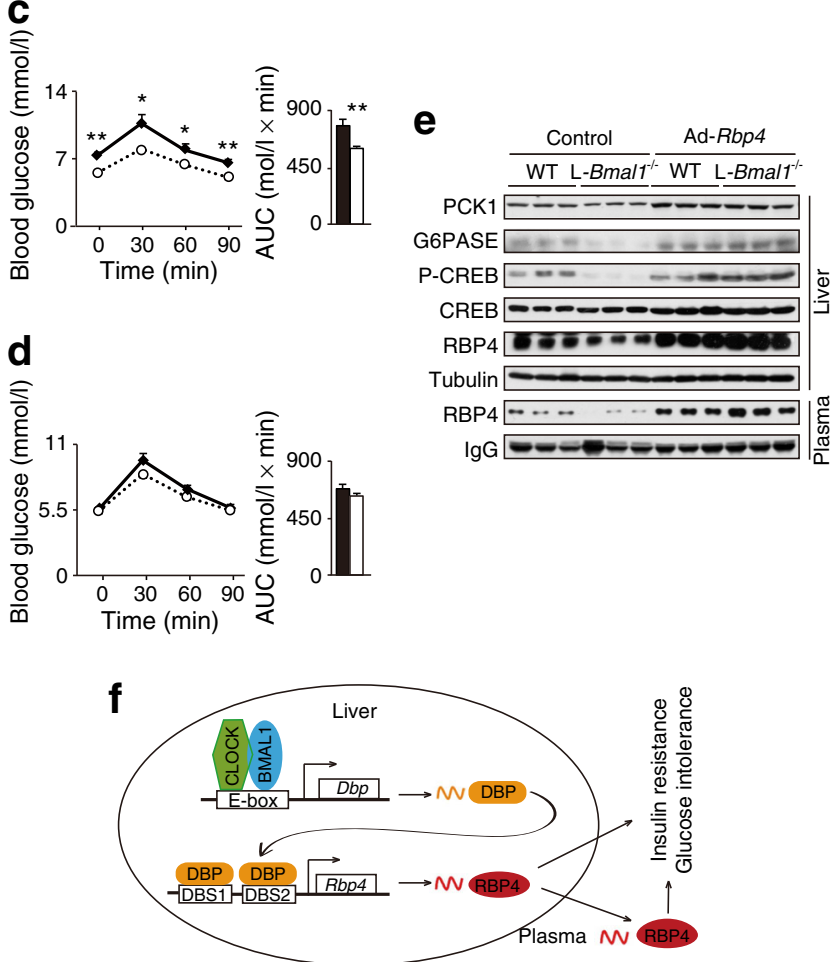

Fig. 5 Hepatic RBP4 is involved in BMAL1/DBP regulation of glucose metabolism. Immunoblotting, with the antibodies indicated, of: (a) WT or Rbp4-null mice (white bars, GFP; black bars, DBP); (b) WT mouse primary hepatocytes infected with indicated adenoviruses (white bars, USi; black bars, Dbpi). Hepatocytes were synchronised by dexamethasone $(1 \mu \mathrm{mol} / \mathrm{l})$ for $2 \mathrm{~h}$. Relative protein levels of PCK1 are presented. Values were normalised to HSP90 or $\beta$-actin and are presented relative to control, set at $1 ;{ }^{*} p<0.05$ with Student's $t$ test. Glucose tolerance of WT and L-Bmall ${ }^{-/-}$mice before (c) and after (d) the injection of Ad-Rbp4 $(n=7-8)$ at ZT8. AUCs were measured. Black diamonds and bars, WT; white circles and bars, L-Bmal1 ${ }^{-1-} ;{ }^{*} p<0.05$ and ${ }^{* *} p<0.01$ with one-way ANOVA. (e) Immunoblotting analysis in the liver homogenates or plasma of WT and L-Bmall ${ }^{-/}$mice, with or without injection of Ad-Rbp4 $(n=3)$. Mice were fed ad libitum and were killed at ZT8. (f) Schematic diagram depicting the circadian regulation of RBP4 by BMAL1 through DBP, which contributes to insulin resistance and glucose intolerance in mice. Data are presented as mean \pm SEM

Meanwhile, several studies have found no correlation between adipose $R b p 4$ mRNA expression and serum protein level [9, 11, 37, 38]. Moreover, Rbp4 mRNA amounts are selectively decreased in the liver, but not in the white adipose tissue, of insulin-sensitive L-Bmal1 ${ }^{-1}$ mice (Fig. 1a and ESM Fig. 1a). Indeed, accumulation of non-secretory RBP4 in the liver impairs glucose tolerance by inducing gluconeogenic genes (ESM Fig. 5b-g), which suggests that the metabolic actions of endogenous RBP4 within the liver also contribute to the salutary phenotype of RBP4-knockdown mice. Together, our findings highlight the importance of the hepatic RBP4 for systemic glucose metabolism.

Previous studies have reported that mice exhibit diurnal variations in insulin sensitivity: the highest insulin sensitivity of mice was found during the night and the lowest was found during the daytime $[39,40]$. Mice with an L-Bmall ${ }^{-/-}$genotype exhibit a time-of-day-dependent insulin-sensitive phenotype [21]. While it has been shown that Glut2 (also known as Slc2a2) has lost its circadian expression in L-Bmall ${ }^{-/-}$mice, the mechanism by which BMAL1 exerts its regulatory function remains largely unknown. Our data support the hypothesis that the insulin hypersensitivity of $\mathrm{L}-\mathrm{Bmall}^{-/}$mice could be due to a deficit of RBP4 (Fig. 5c-e). Although DBP has been reported to be potentially involved in the regulation of cholesterol metabolism [41], its role in glucose homeostasis has not been investigated. In our study, we demonstrate that both DBP and RBP4 are required for the normal circadian changes in insulin sensitivity (Figs 3 and 4). The results of decreased hepatic and circulating RBP4 expression in DBP-knockdown mice, along with the similarity in phenotypes of RBP4- and DBP-deficient mice, suggest that DBP may exert its metabolic action through an RBP4-dependent mechanism. Indeed, the ability of DBP to induce PCK1 expression is compromised by RBP4 deficiency and the effect of DBP knockdown on PCK1 protein is abolished by RBP4 adenoviruses (Fig. 5a, b). Furthermore, hepatic overexpression of RBP4 reverses the insulin-sensitive phenotype of L-Bmal1 ${ }^{--}$mice and restores the expression of gluconeogenic genes (Fig. 5c-e), which strongly implicates a key role of RBP4 in the mechanism by which the circadian clock maintains glucose homeostasis (Fig. $5 \mathrm{f}$ ).

Taken together, our results identify $R b p 4$ as a novel gene under circadian clock control and provide mechanistic insight into its circadian regulation. Furthermore, our results support the prominent role of hepatic RBP4 in the maintenance of metabolic control, and confirm a time-dependent association between RBP4 and insulin resistance in mice. Importantly, the time-of-day-dependent effect of RBP4 on insulin sensitivity is of potential importance in the clinical prognosis of individuals with type 2 diabetes.

Acknowledgements We thank Y. Liu (College of Life Sciences, Wuhan University, Wuhan, China) for generously providing plasma RBP4 antibody. We thank Z. Qian, F. Dang, Q. Xu and R. Wu (Key Laboratory of Nutrition and Metabolism, Institute for Nutritional Sciences, Shanghai Institutes for Biological Sciences, Chinese Academy of Sciences, Shanghai, China) for assisting with experiments. 
Funding This work was supported by grants from the National Basic Research 973 (NBR973) Program (2012CB524900) and (2014CB910500) and the National Natural Science Foundation of China (81390351, 31222028 and 31200891).

Duality of interest The authors declare that there is no duality of interest associated with this manuscript.

Contribution statement YL conceptualised, and together with YL, XM and ZZ, designed the study. XM, ZZ, YQC and YTW were involved in the experimentation and data analysis. All authors contributed to drafting the article or revising it critically for intellectual content. All authors gave final approval of the version to be published. YL is responsible for the integrity of the work as a whole.

\section{References}

1. Quadro L, Blaner WS, Salchow DJ et al (1999) Impaired retinal function and vitamin A availability in mice lacking retinol-binding protein. EMBO J 18:4633-4644

2. Blaner WS (1989) Retinol-binding protein: the serum transport protein for vitamin A. Endocr Rev 10:308-316

3. Newcomer ME, Ong DE (2000) Plasma retinol binding protein: structure and function of the prototypic lipocalin. Biochim Biophys Acta 1482:57-64

4. Yang Q, Graham TE, Mody N et al (2005) Serum retinol binding protein 4 contributes to insulin resistance in obesity and type 2 diabetes. Nature 436:356-362

5. Graham TE, Yang Q, Bluher M et al (2006) Retinol-binding protein 4 and insulin resistance in lean, obese, and diabetic subjects. New Engl J Med 354:2552-2563

6. Cho YM, Youn BS, Lee H et al (2006) Plasma retinol-binding protein-4 concentrations are elevated in human subjects with impaired glucose tolerance and type 2 diabetes. Diabetes Care 29: 2457-2461

7. Kloting N, Fasshauer M, Dietrich A et al (2010) Insulin-sensitive obesity. Am J Physiol Endocinool Metab 299:E506-E515

8. Kotnik P, Fischer-Posovszky P, Wabitsch M (2011) RBP4: a controversial adipokine. Eur J Endocrinol 165:703-711

9. Vitkova M, Klimcakova E, Kovacikova M et al (2007) Plasma levels and adipose tissue messenger ribonucleic acid expression of retinol-binding protein 4 are reduced during calorie restriction in obese subjects but are not related to diet-induced changes in insulin sensitivity. J Clin Endocrinol Metab 92:2330-2335

10. von Eynatten M, Lepper PM, Liu D et al (2007) Retinol-binding protein 4 is associated with components of the metabolic syndrome, but not with insulin resistance, in men with type 2 diabetes or coronary artery disease. Diabetologia 50:1930-1937

11. Yao-Borengasser A, Varma V, Bodles AM et al (2007) Retinol binding protein 4 expression in humans: relationship to insulin resistance, inflammation, and response to pioglitazone. J Clin Endocrinol Metab 92:2590-2597

12. Stefan N, Schick F, Hennige AM et al (2007) High circulating retinol-binding protein 4 is associated with elevated liver fat but not with total, subcutaneous, visceral, or intramyocellular fat in humans. Diabetes Care 30:1173-1178

13. Green CB, Takahashi JS, Bass J (2008) The meter of metabolism. Cell 134:728-742

14. King DP, Takahashi JS (2000) Molecular genetics of circadian rhythms in mammals. Annu Rev Neurosci 23:713-742
15. Lowrey PL, Takahashi JS (2004) Mammalian circadian biology: elucidating genome-wide levels of temporal organization. Annu Rev Genomics Hum Genet 5:407-441

16. Morse D, Sassone-Corsi P (2002) Time after time: inputs to and outputs from the mammalian circadian oscillators. Trends Neurosci 25:632-637

17. Reppert SM, Weaver DR (2002) Coordination of circadian timing in mammals. Nature 418:935-941

18. Brown SA, Kowalska E, Dalmann R (2012) (Re)inventing the circadian feedback loop. Dev Cell 22:477-487

19. Dibner C, Schibler U, Albrecht U (2010) The mammalian circadian timing system: organization and coordination of central and peripheral clocks. Annu Rev Physiol 72:517-549

20. Feng D, Lazar MA (2012) Clocks, metabolism, and the epigenome. Mol Cell 47:158-167

21. Lamia KA, Storch KF, Weitz CJ (2008) Physiological significance of a peripheral tissue circadian clock. Proc Natl Acad Sci U S A 105:15172-15177

22. Rudic RD, McNamara P, Curtis AM et al (2004) BMAL1 and CLOCK, two essential components of the circadian clock, are involved in glucose homeostasis. PLoS Biol 2:1893-1899

23. Chen JL, Zhang W, Lin JY et al (2014) An efficient antiviral strategy for targeting hepatitis $\mathrm{b}$ virus genome using transcription activator-like effector nucleases. Mol Ther 22:303-311

24. He TC, Zhou S, da Costa LT, Yu J, Kinzler KW, Vogelstein B (1998) A simplified system for generating recombinant adenoviruses. Proc Natl Acad Sci U S A 95:2509-2514

25. Becker TC, Noel RJ, Coats WS et al (1994) Use of recombinant adenovirus for metabolic engineering of mammalian cells. Methods Cell Biol 43:161-189

26. Sun X, Dang F, Zhang D et al (2015) Glucagon-CREB/CRTC2 signaling cascade regulates hepatic BMAL1 protein. J Biol Chem 290:2189-2197

27. Ramanathan C, Khan SK, Kathale ND, Xu H, Liu AC (2012) Monitoring cell-autonomous circadian clock rhythms of gene expression using luciferase bioluminescence reporters. J Vis Exp. doi: $10.3791 / 4234$

28. Ramanathan $\mathrm{C}, \mathrm{Xu} \mathrm{H}$, Khan SK et al (2014) Cell type-specific functions of period genes revealed by novel adipocyte and hepatocyte circadian clock models. PLoS Genet 10:e1004244

29. Sato TK, Yamada RG, Ukai H et al (2006) Feedback repression is required for mammalian circadian clock function. Nat Genet 38: 312-319

30. Mauvoisin D, Wang JK, Jouffe C et al (2014) Circadian clockdependent and -independent rhythmic proteomes implement distinct diurnal functions in mouse liver. Proc Natl Acad Sci U S A 111:167-172

31. Canaple L, Rambaud J, Dkhissi-Benyahya O et al (2006) Reciprocal regulation of brain and muscle Arnt-like protein 1 and peroxisome proliferator-activated receptor alpha defines a novel positive feedback loop in the rodent liver circadian clock. Mol Endocrinol 20:1715-1727

32. Ripperger JA, Schibler U (2006) Rhythmic CLOCK-BMAL1 binding to multiple E-box motifs drives circadian Dbp transcription and chromatin transitions. Nat Genet 38:369-374

33. Mueller CR, Maire P, Schibler U (1990) DBP, a liver-enriched transcriptional activator, is expressed late in ontogeny and its tissue-specificity is determined posttranscriptionally. Cell 61:279291

34. Abel ED, Peroni O, Kim JK et al (2001) Adipose-selective targeting of the GLUT4 gene impairs insulin action in muscle and liver. Nature 409:729-733

35. Tsutsumi C, Okuno M, Tannous L et al (1992) Retinoids and retinoid-binding protein expression in rat adipocytes. J Biol Chem 267:1805-1810 
36. Kloeting N, Graham TE, Berndt J et al (2007) Serum retinolbinding protein is more highly expressed in visceral than in subcutaneous adipose tissue and is a marker of intra-abdominal fat mass. Cell Metab 6:79-87

37. Janke J, Engeli S, Boschmann M et al (2006) Retinol-binding protein 4 in human obesity. Diabetes 55:2805-2810

38. Kos K, Wong S, Tan BK et al (2011) Human RBP4 adipose tissue expression is gender specific and influenced by leptin. Clin Endocrinol 74:197-205
39. Sadacca LA, Lamia KA, deLemos AS, Blum B, Weitz CJ (2011) An intrinsic circadian clock of the pancreas is required for normal insulin release and glucose homeostasis in mice. Diabetologia 54: 120-124

40. Li MD, Ruan HB, Hughes ME et al (2013) O-GlcNAc signaling entrains the circadian clock by inhibiting BMAL1/CLOCK ubiquitination. Cell Metab 17:303-310

41. Wuarin J, Falvey E, Lavery D et al (1992) The role of the transcriptional activator protein DBP in circadian liver gene expression. $\mathrm{J}$ Cell Sci 16:123-127 